\title{
Few-Layer Graphene Modified by Bridge-Like Defects as Li-Storing Cells
}

\author{
Arkady M. Ilyin \\ National Nanolaboratory, Kazakh National University, Almaty, Kazakhstan
}

Received: June 12, 2014 / Accepted: June 23, 2014 / Published: June 25, 2014.

\begin{abstract}
The paper presents some results of computer simulations and density functional theory calculations of the energetic and structural characteristics of few-layer graphene nanostructures as storing cells for Li. Modeling and calculations allow to reveal some peculiarities in such systems, which can be considered as causes of known dimension instabilities and shortening of the life-time of Libased electrical power devices. Results of the computational study and calculations predict, that all these problems can be in significant measure solved by modifying of few-layer graphene nanostructures by bridge -like defects, which make them much more stiffer and stable against the deformation.
\end{abstract}

Key words: Lithium power sources, few-layer graphene, bridge-like defect, computer simulation.

\section{Introduction}

It is well known, that graphite structures are currently considered as materials for electrodes in lithium based power devices, mainly due to their high reversibility, low weight and low operating potential. Recently nanomaterials based on thin graphite particles have found a use in the technological field relating to production of lithium-ion rechargeable batteries $[1,2]$. But there are some obstacles in the progress in this field. One of the main problems relates to dimension instability of battery elements, caused by large possible deformation or even fracture of electrode materials due to charge, discharge processes $[3,4]$. Another peculiarity displays by using relatively large graphite particles in which time of lithium diffusion by intercalation and de-intercalation processes can be large, that significantly decreases the effectiveness of operating power devices [5]. It originates from restricted by two-dimensions lithium transportation in defect-free flaky graphite structures. Seemingly, the

Corresponding author: Arkady M. Ilyin, doctor of science, professor, research fields: nanomaterials and eletron spectroscopy. E-mail: ilyinar@mail.ru. physical situation can become much better by using ultra thin graphite structures with nano-scaled dimensions (few-layer graphene, FLG). But there is an obvious lack of studies of Li-FLG nanostructures, especially by ab-initio approach. In particular, many of physical and structural characteristics of FLG are not well understood yet. Therefore, these materials become objects of intensive experimental and theoretical studies. This paper presents results of computer simulation and density functional theory (DFT) calculations $[6,7]$ of some possible configurations of nanoscaled FLG cells intercalated with lithium. Additionally, effect of structural defects like vacancies and bridge-like defects (BLD) [8-10] on mechanical properties was studied.

\section{Simulations and Calculations}

\subsection{Simulations of Structural Characteristics of Li-FLG Cells}

In order to perform modeling more adequate to real carbon nanostructures, all DFT calculations in this work were performed by using energy optimization procedure $[6,7]$. At the first stage of simulations we 
considered possible stable configurations of $\mathrm{Li}$ on ideal graphene and calculated their energetic characteristics (Fig. 1). Calculations gave for all these configurations very low values of the binding energies of Li: nearly $0.1 \mathrm{eV}$.

Therefore, the next step of simulations was to study possible configurations with Li linked to structural defects in graphene. Fig. 2a presents a single Li atom connected with a graphene's vacancy. In this case the bonding energy is as large as $0.4 \mathrm{eV}$, and $\mathrm{Li}$ atom after energy optimization occupies an asymmetrical position with the distance $0.9 \AA$ from the graphene's sheet. In Fig. $2 \mathrm{~b}$ one can see a more complex defect, involving a vacancy with a Li-dumbbell, attached to it. In this case the binding energy was as large as $0.6 \mathrm{eV}$. Obviously, these kinds of defects should be taken into account when considering possible practical usage of FLG as $\mathrm{Li}$ storing cells. Fig. 3 presents a computational models of two-layer cells containing Li. One can see obvious change of cells shape (blister-like deformation ), increasing with the level of lithiation. This effect was revealed after using the energy optimization procedure. The maximum deformation (the distance between blister tops) in the case for 10 , cluster was about $12 \%$ with the average area occupied per $\mathrm{Li}$ atom approximately $3 \AA^{2}$. Obviously one can see a tendency to clusterization of Li within the cells. Obviously, it was of a great importance, to determine a maximum local change of size of cells in dependence on Li cluster size by given size of cells. In our computational experiments, every two-layer graphene cell involved 112 carbon atoms.

Fig. 4 illustrates very essential effect of BLD on keeping the perpendicular size of two-layer cells nearly as that in pristine state. One can see, that the cell, modified by BLD shows no signs of noticeable deformation. Moreover, it should be noticed, that BLD draws together graphene sheets and the distance between them in the area close to the defect is smaller than in pristine cells.

Graph in Fig. 5 illustrates dependence of the maximum deformation (top-to-top distance) of two-layer graphene cells on number of $\mathrm{Li}$ atoms with and without BLD. It can be seen that BLD essentially modify stiffness of two-layer graphene cells.

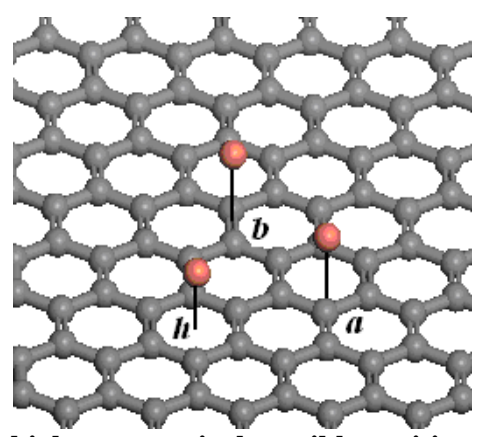

Fig. 1 The high-symmetrical possible positions of $\mathrm{Li}$ atoms on graphene surface: $h$ is over the center of a hexagon; $a$ is over a carbon atom; $b$ is over the center of the $C-C$ bond.

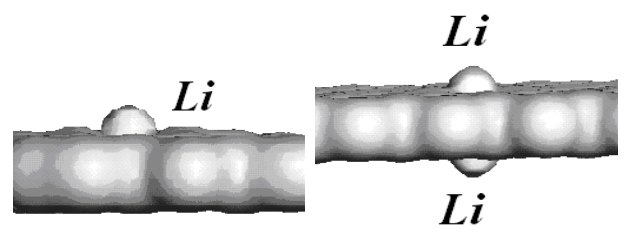

(b)

Fig. 2 Computational models of possible configurations of $\mathrm{Li}$ - graphene composition: (a) a single $\mathrm{Li}$ atom-vacancy ; (b) Li-Li dumbbell-vacancy. The both images illustrate the distribution of electron charge at the density as large as $0.2 \mathrm{el} / \AA^{3}$.

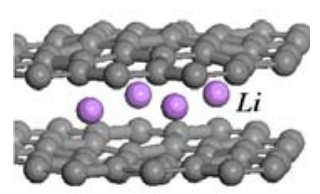

(a)

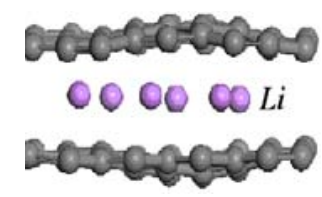

(b)

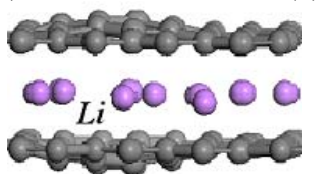

(c)

Fig. 3 Li inside a 2-layer graphene cell: $N=$ (a) 4; (b) 6 and (c) 10 .

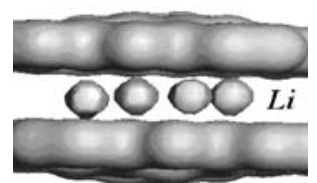

(a)

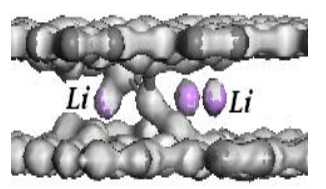

(b)
Fig. 4 The distribution of the electron charge in two-layer cells containing $\mathrm{Li}$ : (a) a pristine state and (b) modified by BLD. 
Fig. 6 presents results of simulation of three-layer cells containing Li. Fig. 6a illustrates simulations of three- layer storing cells with large enough numbers of $\mathrm{Li}$ in betweens $(\mathrm{N}=10+10)$. One can see very large change of size in the center (top-to-top) of the cell. Fig. $6 \mathrm{~b}$ presents three-layer cell containing the same number of lithium, but with BLD between the two upper sheets. One can see the obvious effect of decreasing the top layer deformation. Obviously, the level of the perpendicular size changes is much larger for sub-cell between lower layers. Fig. $6 \mathrm{c}$ presents the electron charge distribution at $0.4 \mathrm{el} / \AA^{3}$ proves that $\mathrm{Li}$ inside the cells remains in chemically free state. Moreover, one can see, that in general the level of deformation for three layer graphene structures noticeably lower than for two-layer cells.

\subsection{Simulations of Li Diffusion in FLG}

One more important characteristic of lithium-ion devices is the effective mobility of $\mathrm{Li}$ in the structure of batteries. Therefore the study of diffusion motion of $\mathrm{Li}$ in FLG cells is of great interest. Fig. 7 represents results of simulations and calculations for motion of $\mathrm{Li}$ in the structure and near edges of two-layer cells. Fig. 7a presents four possible motion directions of high symmetry.

During working of batteries lithium diffuses into and out of the electrodes. Fig. 7 illustrates energetic potential barriers for lithium going out of a two-layer cell in directions 1-3 with the initial point in the center of a hexagon near the edge of cell and into cell structure (direction 4). In defect-free graphite lithium motion is restricted to two-dimensional diffusion because lithium

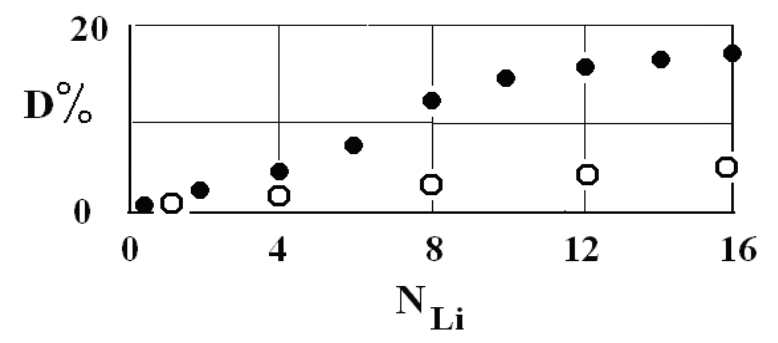

Fig. 5 The Li quantity dependences of deformation for two-layer cells. Black circles: without BLD; weight circles: modified by BLD.

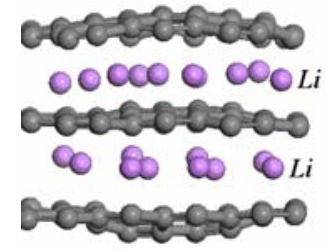

(a)

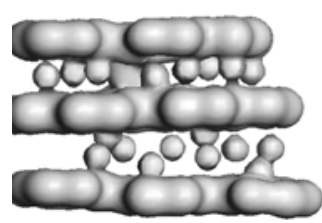

(c)

Fig. 6 Modeling of three -layer cells filled with $\mathrm{Li}$. (a) 10 + 10; (b) modified by BLD $10+10$ and (c) electron charge distribution for the (b) configuration.

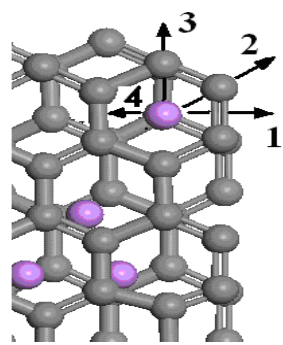

(a)

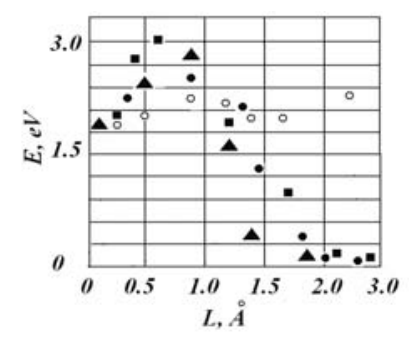

(b)
Fig. 7 Potential barriers calculated for motion of lithium near edges of a two-layer cell in the three different directions (shown by arrows) : (1) black circles, (2) squares, (3) triangles and (4) empty circles.

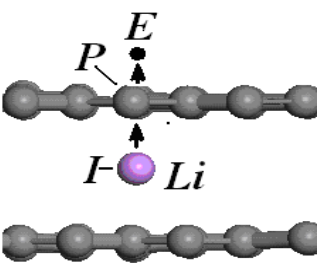

(a)

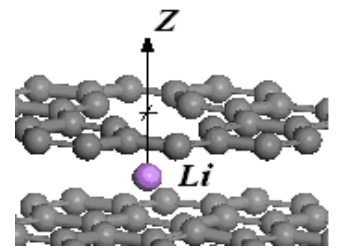

(c)

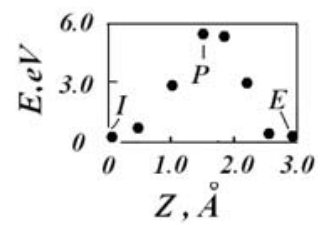

(b)

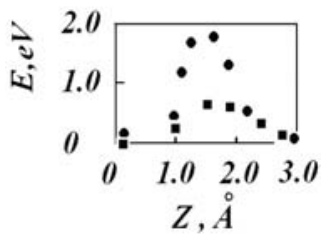

(d)
Fig. 8 Simulation of possible ways of motion of lithium through the graphene plane in two-layer graphene cell: (a) modeling of supposed way of $\mathrm{Li}$ passing out through hexagon of an ideal graphene sheet; (b) the calculated potential energy barrier for this direction; (c) a possible calculated configuration of an intercalated Li-vacancy complex with a possible exit direction of $\mathrm{Li}$ through the vacancy and (d) the energy barriers for a passing $\mathrm{Li}$ through vacancy (circles) or bi-vacancy (squares). 
jumping through a carbon honeycomb is energetically impossible. Obviously, it increases time of lithium diffusion motion by intercalation and de-intercalation processes and in combination with small atomic relation of $\mathrm{Li} / \mathrm{C}$ significantly decreases the effectiveness of operating power devices. Our simulations show, that in FLG structural defects like vacancies possibly create additional ways of passage of lithium and in general, its mobility will be increased. Fig. 8 represents the results of calculations shown possible perpendicular directions of passage of lithium through a graphene sheet: Fig. 8a shows a scheme of supposing motion of Li through a hexagon structure; Fig. $8 \mathrm{~b}$ shows a high potential barrier for this direction; Fig. $8 \mathrm{c}$ shows the atomic structure of simple vacancy-like structural defect. Calculations show that potential barriers on the way of Li passing through a single vacancy (black marks) or bi-vacancy (empty marks) are comparable with that at the edge of cell and much more less than that for ideal graphene sheet (Fig. $8 b)$. The zero point of $Z$ axis is placed in initial position of $\mathrm{Li}$ atom as epicenter of a hexagon in the middle plain between graphene sheets. These results prove that lithium diffusivity in FLG can be essentially increased due to production of structural defects.

\section{Conclusions}

Computer simulations and quantum mechanical calculations of structural and energy characteristics of single and few-layer graphene nanocells containing lithium have been performed. Results of modeling showed that nanoscaled few-layer graphene are promising systems of interest as storing cells of lithium with light weight and large capacity. It was revealed that FLG cells, modified by bridge-like structural defects can provide very high level of strength and stiffness with minimizing the dimension instability. Moreover, the existence of vacancies and bridge-like defects enlarges the permeation and mobility of Li through graphene planes. Results of the study allow predict, that radiation modification of few -layer graphene might become a key technology in their application as lithium storing cells in production of stable and save lithium based power sources.

\section{References}

[1] R.A. Huggins. Advanced Batteries, Materials Science Aspects, Springer, New York, 2009, p. 459.

[2] G. Kuchinskis, G. Bajars, J. Kleperis, Graphene in lithium ion battery cathode materials: A review, Journal of Power Sources 240 (2013) 66-79.

[3] K. Zhao, M. Pharr, Sh. Cai, J.J. Vlassak, Zh. Suo. Large plastic deformation in high-capacity lithium ion batteries caused by charge and discharge, J. Am. Ceram. Soc. 94 (2011) S226-S235.

[4] Y.H. Hu, X.H. Zhao, Z.G. Suo. Averting cracks caused by insertion reaction in lithium ion batteries, J. Mater. Res. 25 (6) (2010) 1007-1010.

[5] K. Persson, V.A. Sethuraman, L.J. Hardwik, Y. Hinuma. Lithium diffusion in graphitic carbon, Journal of Physical Chemistry Letters 1 (8) (2010) 1176-1180.

[6] B. Delley, An all-electron numerical method for solving the local density functional, J. Chem. Phys. 92 (1990) 508-517.

[7] B. Delley, From Molecules to Solids with the $\mathrm{DMol}_{3}$ Approach, J. Chem. Phys. 113 (2000) 7756-7764.

[8] A.M. Ilyin, R.R. Nemkaeva, V.V. Kudrjashov. Computational study of graphene- and few layer graphene-metal composites induced by radiation, Journal of Materials Science \& Engineering B 3 (3) (2013) 161-166.

[9] A.M. Ilyin, G.W. Beall, I.A. Tsyganov, Simulation and study of bridge-like radiation defects in the carbon nano-structures. J. Comp. Theor. Nanosci. 7 (10) (2010) 2004-2007.

[10] A.M. Ilyin, Computer simulation of radiation defects in graphene and relative structures, Graphene Simulation, Gong, InTech: Rijeka, CR, 2011, pp. 39-54. 